\title{
Multilinguales
}

Multilinguales

12 | 2020

Varia 2019

\section{Espace, mythe et histoire dans Un oued, pour la mémoire de Fatéma Bakhaï}

space, myth and history in Un oued, pour la mémoire by Fatéma Bakhaï

\section{Brahim Ait Amokrane}

\section{OpenEdition}

Journals

Édition électronique

URL : https://journals.openedition.org/multilinguales/4227

DOI : 10.4000 /multilinguales.4227

ISSN : 2335-1853

Éditeur

Université Abderrahmane Mira - Bejaia

Référence électronique

Brahim Ait Amokrane, «Espace, mythe et histoire dans Un oued, pour la mémoire de Fatéma Bakhaï », Multilinguales [En ligne], 12 | 2020, mis en ligne le 14 février 2010, consulté le 30 juin 2021. URL : http:// journals.openedition.org/multilinguales/4227 ; DOI : https://doi.org/10.4000/multilinguales.4227

Ce document a été généré automatiquement le 30 juin 2021.

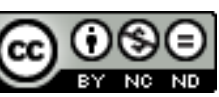

Multilinguales est mise à disposition selon les termes de la Licence Creative Commons Attribution -

Pas d'Utilisation Commerciale - Pas de Modification 4.0 International 


\title{
Espace, mythe et histoire dans Un oued, pour la mémoire de Fatéma Bakhaï $^{1}$
}

\author{
Space, myth and history in Un oued, pour la mémoire by Fatéma Bakhaï
}

Brahim Ait Amokrane

1 Voici une douzaine d'années que la production littéraire de Fatéma Bakhaï est objet d'étude et de recherche, en Algérie et ailleurs : elle est analysée dans ses rapports à l'Histoire, à la question féminine ainsi qu'à la notion d'identité, qui demeure chère à toute la littérature maghrébine. Mais il est utile de rappeler que c'est en 1990 que cette écrivaine prolifique vient à l'écriture, avec un roman planté dans le décor du vieil Oran : La Scaléra. Dès ce premier roman, on voit s'introduire dans le récit un énorme appareil historique, qui correspond à un goût avoué de l'auteure pour la connaissance du passé :

Il faut écrire l'Histoire, la raconter, la peindre, la filmer, la jouer, la figurer, la penser, la mythifier pour en découvrir le sens. [....] L'Histoire est plus palpable, plus vraie, plus proche racontée par un film ou un roman. Chaque détail est important pour nourrir l'imaginaire et c'est ce dont les Algériens ont besoin pour ne plus, stupidement, se surestimer parfois, se dévaloriser souvent. [ ...] Les Algériens doivent mettre en valeur leur héritage sans rien dilapider, se construire, apaisés, et s'unir, confiants, en s'appuyant sur un passé qui n'appartient qu'à eux (2012: 8-9).

C'est ainsi que Bakhaï justifie son engouement pour l'Histoire de son pays. Dans le contexte actuel de "guerre des mémoires " (Stora, 2007), ses propos sont plus que jamais d'actualité. Car, le retour au passé devrait aider à mieux faire face aux défis du présent. Or les lieux de mémoire sont d'un intérêt particulier à cet égard, puisque, comme l'explique Pierre Nora, » les lieux sont notre moment de l'histoire nationale » (1984: 30). D'où l'intérêt que leur porte Bakhaï, dans ses œuvres.

2 Afin d'explorer les dimensions poétique, symbolique et identitaire de ce type d'espace, cet article examinera l'usage qu'elle en a fait, dans son roman - qui n'en est pas vraiment $u^{2}$ - Un oued, pour la mémoire, publié en 1995. Dans un souci de précision, il faut dire que cette date correspond à un moment crucial de l'Histoire algérienne, étant 
donné que "l'année 1995 marque un tournant dans la violence [terroriste], avec la multiplication d'attentats à la voiture piégée dans les grandes ville du pays » (Stora, 2001 : 30). Néanmoins, à la différence de la quasi majorité des auteurs de sa génération, Fatéma Bakhaï ne s'inscrit pas dans la littérature dite de «l'urgence »; toute sa pratique romancière va à la quête de la mémoire, " pour donner du sens à la tragédie post-indépendance, de la traquer là même où le témoignage brut ou romancé s'avère impuissant » (Mokhtari, $2006: 31$ ).

Dans la droite ligne de raisonnement, Un oued, pour la mémoire peut se lire comme une longue analepse, à travers laquelle le lecteur revisite le passé d'Oran. Le texte retrace, en effet, dans une perspective rétroactive, l'histoire d'un vieil immeuble bâti par des colons, sur oued souterrain qui n'est autre que l'oued de Djaffar, le fondateur de la ville, et ses deux lionceaux.

Monsieur Weber, l'architecte, est jusqu'à sa mort tenaillé par le regret d'avoir «pu se résoudre à couler son béton sur un oued vivant ", pour obéir au rêve de madame Boissier, qui ne voulait pas tenir compte du danger qu'il y avait à bâtir son immeuble à cet endroit. Au fil des ans, la maison connaît plusieurs occupants, mais laisse peu à peu transparaître les signes de la présence souterraine de l'oued, qui lutte pour revoir la lumière. $\mathrm{Au}$ dénouement de l'œuvre,environ cent ans après sa construction, l'immeuble s'effondre, suite aux pluies diluviennes qui se sont abattues sur la ville. Toutes les sources, même celles dont personne ne se souvient, rejaillissent aux endroits les plus inattendus. Ainsi, l'oued occulté reprend sa place, au mépris de tous ceux qui l'ont ignoré.

Nous pouvons d'ores et déjà noter qu'Un oued, pour la mémoire oppose deux espaces : le vieil immeuble construit par les colons et l'oued lié à l'ancêtre fondateur. L'effondrement total de l'un, ou sa destruction par les eaux, et la résurgence de l'autre donnent au récit de forts accents mythiques, dans la mesure où ces faits symbolisent la Fin du Monde et la cosmogonie. Eliade explique que "l'eschatologie n'est que la préfiguration d'une cosmogonie de l'avenir. Mais toute eschatologie insiste sur ce fait : que la Nouvelle Création ne peut avoir lieu avant que ce monde-ci ne soit définitivement aboli» (1975: 69). C'est cette émergence du mythe de la Fin qui retient le plus notre intérêt, dans le texte. La question que nous nous posons est la suivante : quels sont les éléments scripturaux qui attestent de cette émergence, et comment celle-ci se relie-telle avec les espaces représentés?

D’après Eliade, la Fin du Monde n'est pas imaginée de la même façon par tous les primitifs. Mais «les mythes du Déluge sont les plus nombreux, et presque universellement connus [...]. À côté des mythes diluviens, d'autres relatent la destruction de l'humanité par des cataclysmes de proportions cosmiques : tremblement de terre, incendies, écroulement de montagnes, épidémies, etc.» (1975: 71). Nous aurons l'occasion de montrer, dans la suite de cet article, que certaines de ces images apocalyptiques sont présentes dans Un oued, pour la mémoire, mais nous comptons surtout démontrer que c'est la doctrine indienne des quatre Âges du monde, c'est-àdire : l'éternelle création, détérioration progressive, anéantissement et re-création de l'Univers (Eliade, 1975: 81) qui rythme tout le récit. Pour ce faire, nous nous intéresserons d'abord à l'émergence du mythe de l'Âge d'or dans le texte. Ensuite, nous démontrerons que la détérioration progressive du Monde se traduit par la disparition de l'oued et la lente dégradation de l'immeuble. Enfin, nous étudierons comment le 
"Déluge ", qui achève le processus de détérioration, ouvre la voie à la fois à une recréation du Monde et à une récupération de l'état paradisiaque.

\section{Le paradis primoridial ou les origines mythiques d'Oran}

5 La théorie indienne des Âges du Monde comprend le mythe de la perfection des commencements ou ce que l'on appelle l'Âge d'or. D'autres civilisations (les Babyloniens, les Grecs, etc.) connaissaient également le mythe d'un Paradis primordial. Mais l'essentiel des croyances s'y rapportant peuvent être résumées de la sorte: les hommes vivaient en parfaire harmonie avec la Nature ; ils comprenaient le langage des animaux et vivaient avec eux en paix ; ils ne travaillaient point et trouvaient, à portée de main, une nourriture abondante (Eliade, 1990 : 78).

Les pays de l'Âge d'or sont désormais des contrées inaccessibles, certes, mais l'histoire littéraire montre que les écrivains n'ont jamais abandonné le rêve d'un monde restauré dans sa beauté et sa gloire premières. Chez Bakhaï, la recherche de la "Terre-sansmal» (Eliade, $1971: 170)$ se rattache au récit de la fondation d'Oran par un Andalou nommé Djaffar. L'histoire de cet ancêtre mythique est rapportée par le grand-père d'Aïcha, qui la transmet, à son tour, à sa petite-fille, Mounia :

« - je t'en prie grand-père, que Dieu te garde, raconte-moi, raconte-moi ! »

Alors le vieil homme, rassuré, s'éclaircissait la voix et lissant ce qui lui restait de

barbe entreprenait de renouer avec ces temps anciens que la petite-

fille, éblouie, imaginait comme un âge d'or dont elle serait à jamais exclue (2002 :

17).

6 L'arrivée de Djaffar à Oran est placée sous le signe du destin, puisqu'en fuyant Cordoue pour échapper à un mariage forcé,il embarqua sur un bateau, en partance pour l'Egypte et non pour l'Algérie. Soudain, une terrible tempête survint et causa la destruction du navire. Djaffar et ses compagnons étaient encore loin d'Alexandrie, mais "ils aimèrent cette terre où la destinée les avait conduits et ils décidèrent d'y rester » (2002: 19) :

Ainsi donc, Djaffar se retrouvait avec ses compagnons dans un pays dont ils ne connaissaient pas encore le nom et qui semblait désert, car pas une seule habitation ne s'élevait sur cette plaine pourtant accueillante. [...] Ils marchèrent longtemps, croisèrent des cavernes et les nomades leur indiquèrent un endroit où des sources claires et douces, dans une vallée aux fruits abondants, leur permettraient le repos dont ils semblaient avoir besoin (2002:19).

Les traits $\mathrm{du}$ mythe de l'Âge d'or sont aisément repérables dans ce passage : l'abondance spontanée de la Terre, les arbres fruitiers, les sources claires et douces, le repos et la paix. Tous ces éléments concourent à dessiner l'image mythique d'une Nature primordiale, une Nature féconde et maternelle, dans laquelle ont vécu les Ancêtres.

7 L'amitié avec les animaux et la connaissance de leur langage attestent également de cette réintégration de la condition de l'homme primordial dont nous parlent les mythes paradisiaques :

Le lendemain, au moment de son bain, les lionceaux étaient encore là, et il en alla de même les jours suivants mais de lionne, point de trace. C'est alors qu'il se rappela le rêve qu'il avait fait sur le navire quelques heures avant la terrible 
tempête, et il comprit soudain que les deux lionceaux lui portaient un message, un signe du destin qu'il devait accepter.

Il lui sembla, ce jour-là, que les lionceaux l'observaient avec dans le regard comme un appel muet mais si clair que sans plus réfléchir il s'avança vers eux. Les lionceaux, satisfaits, d'un même mouvement s'engagèrent dans les fourrés en se retournant de temps en temps comme pour s'assurer que l'homme les suivait bien. Et puis, derrière un amas de grosses pierres grises, ils s'arrêtèrent soudain en poussant des petits cris. Djaffar s'approcha le cœur battant et découvrit la lionne allongée sur le flanc, les pattes raides et les yeux grands ouverts. Elles était morte, victime sans doute d'un éboulement qu'elle n'avait pas prévu, car des herbes et des branchages encore verts apparaissaient sous les blocs de rocher.

Djaffar, ému, s'assit sur une pierre plate et les lionceaux sans hésitation vinrent se frotter contre ses jambes.

Ils ne se quittèrent plus jamais (2002: 20).

8 Volontairement long pour nécessité de démonstration, cet extrait nous frappe d'emblée : les lionceaux, petits rois des animaux, se font ici attendrissants et dociles, à tel point que Djaffar décide de ne jamais s'en séparer. Ce fait est d'autant plus fabuleux que les lions font partie des carnivores les plus dangereux pour l'homme. Mais cette amitié entre homme et animal n'est pas une simple évocation d'un passé mythique. C'est le présent de la tragédie que vit le pays - nous sommes en pleine décennie noire - qui suscite la nostalgie d'un nouvel Âge d'or, c'est-à-dire le désir de vivre en dehors de l'Histoire, dans un monde auroral et béatifique, »tel qu'il était avant que le Temps ne le ronge et l'Histoire ne l'avilisse » (Eliade, $184: 1971)$ : «c'est aux époques de crise, quand tout semble basculer, que le mythe [de l'Âge d'or] resurgit » affirme Marie-Josette Bénéjam ( 1988 : 55).

L'extrait que nous avons donné invite également à une réflexion sur le mythe de la fondation d'Oran. Pour ce faire, rappelons que «tout espace sacré implique une hiérophanie, une irruption du sacré qui a pour effet de détacher un territoire du milieu cosmique environnant et de le rendre qualitativement différent » (Eliade, 1988 : 30). Mais, souvent, » il n'est pas même besoin d'une théophanie ou d'une hiérophanie proprement dites: un signe quelconque suffit à indiquer la sacralité du lieu» (Eliade, 1988: 30). Pour le faire comprendre, Eliade rapporte une légende :

Le marabout qui fonda El-Hemel à la fin du XVI siècle s'arrêta pour passer la nuit près de la source et planta un bâton en terre. Le lendemain, voulant le reprendre pour continuer sa route, il trouva qu'il avait pris racine et que des bourgeons avaient poussé. Il y vit l'indice de la volonté de Dieu et fixa sa demeure en cet endroit $(1988: 30)$.

Ici, un signe, » [q]uelque chose qui n'appartient pas à ce monde s'est manifesté d'une manière apodictique et, ce faisant, a tracé une orientation ou a décidé d'une conduite " (Eliade, $1988: 30$ ). Parfois, ce sont les animaux qui révèlent la sacralité du lieu :» On poursuit une bête fauve et, à l'endroit où on l'abat, on élève le sanctuaire; ou bien on lâche en liberté un animal domestique -un taureau, par exemple -, après quelques jours on le sacrifie à l'endroit même. On élèvera ensuite l'autel et on bâtira le village autour de cet autel » (Eliade, $1988: 31$ ).

9 Les hommes, on l'aura compris, ne choisissent pas librement l'emplacement sacré : ils ne font que le chercher et le découvrir à l'aide de signes mystérieux. Dans le cas qui nous occupe, c'est par le biais des lionceaux que Djaffar reçoit la révélation d'un lieu sacré. Ce sont eux qui lui montrent que sa maison doit être construite au bord de l'oued: «il comprit soudain que les deux lionceaux lui portaient un message, un signe de destin qu'il devait accepter» (2002: 20). Comme dans la légende rapportée par Eliade, un signe 
porteur de signification sacrée a révélé un «point fixe», un «Centre» où le personnage peut fonder son habitation :

C'est là, disait le vieillard en désignant l'immeuble de la rue en pente, c'est là que Djaffar a bâti sa maison au bord de l'oued. C'était la première maison de la ville, de notre ville, et tu comprends maintenant pourquoi elle porte son joli nom : Les Deux Lionceaux, Wahran, Oran (2002: 20-21).

En imitant l'acte exemplaire des dieux, Djaffar se détache du temps profane et rejoint magiquement le Grand temps, le Temps sacré des commencements. La naissance de la ville d'Oran peut alors être considérée comme une répétition de la cosmogonie :

Toute histoire mythique relatant l'origine de quelque chose présuppose et prolonge la cosmogonie. Du point de vue de la structure, les mythes d'origine sont homologables au mythe cosmogonique. La création du monde étant la création par excellence, la cosmogonie devient le modèle exemplaire pour toute espèce de «création ». [...] Tout mythe d'origine raconte et justifie une "situation nouvelle » - nouvelle dans la mesure où elle n'était pas là dès le début du Monde. Les mythes d'origines prolongent et complètent le mythe cosmogonique:ils racontent comment le Monde a été modifié, enrichi ou appauvri (Eliade, 1975 : 33-34).

La fonction dévolue aux mythes d'origine est nettement explicative. Le mythe de la fondation d'Oran ne déroge pas à la règle. Mais, creusons plus avant: comment et pourquoi s'effectue le passage du domaine de la réalité historique à celui du mythe ? On peut dire que la mémoire étant par nature sélective, subjective et oublieuse de certains détails, le souvenir des événements historiques se modifie au fil du temps, créant ipso facto les zones d'ombres nécessaires à l'éclosion du mythe: "Tout processus de mythification suppose en effet une plasticité inhérente au personnage, tributaire des lacunes ou du mystère qui lui sont attachés » affirme Pascale Auraix- Jonchière (2008 : 238). Or, s'il ne fait pas de doute qu'Oran a un passé andalou, sa fondation reste marquée par l'énigme : les historiens ignorent le véritable nom de son fondateur et ne proposent, finalement, que des hypothèses quant à son origine. L'étymologie même de son appellation arabe, Wahran, divise les spécialistes de l'onomastique, comme le confirme Farid Benramdane : "Wahran fait partie de cette catégorie de toponymes qui connaissent un nombre important d'interprétations" (2004: 253). Cet enseignant-chercheur précise, néanmoins, que l'une des hypothèses dominantes consiste à dire que cette appellation signifie "Oued des lions» (270), étymologie qui nous renvoie aux deux éléments narratifs essentiels de notre mythe : l'oued de Djaffar et les deux lionceaux dont il ne veut se séparer.

Si la naissance d'Oran constitue en soi un mythe d'origine, l'histoire de son fondateur, Djaffar, fait office d'un mythe tribal, dans le sens prôné par Mohamed Ismaïl Abdoun :

Le mythe tribal tient lieu de "mémoire» du groupe social. Grâce au discours mythique sous formes de légendes, de maximes, poèmes, hagiographie, chroniques familiales, narrations diverses...les membres du groupe sont informés, de génération en génération, de leur histoire. C'est, d'une certaine manière, l'expression de leur personnalité collective (2006:53).

En effet, l'histoire de Djaffar apparait comme création collective : chaque génération a dû apporter sa part, inventer tel ou tel événement, mêlant de façon tellement inextricable l'imagination et la réalité :

Mille ans, un peu plus ou un peu moins, mille ans que notre ancêtre a posé pour la première fois le pied sur ces rivages, mille ans que de génération en génération nous racontons son histoire, et même si notre aïeul aujourd'hui ne s'y reconnaîtrait plus, et bien cela ne fait rien, l'important est de garder dans nos mémoires son souvenir, et si ses aventures ont été transformées, embellies, enrichies par tous les 
grands-pères, toutes les grand-mères qui les ont racontées à leurs petitsenfants, c'est parce que tous l'ont aimé, chacun à sa manière ( $2002: 17)$.

11 À cette étape de notre lecture, nous pouvons dire que le rapport à l'Histoire, dans Un oued, pour la mémoire, est moins mimétique que transfiguratif. L'histoire prétend alors à " une vérité qui lui est propre, éclairée par une pensée et une visée de l'Histoire, découverte et recréée par l'imagination et [...] écoutée aux portes de la légende ou des traditions populaires » (Duchet, $1975: 257-258$ ).

\section{Le vieil immeuble de la rue en pente : une allégorie de la mort}

La doctrine des quatre Âges du monde véhicule l'idée archaïque, et extrêmement répandue, de la dégradation progressive du Cosmos, nécessitant sa destruction et sa recréation périodique (Eliade, $1975: 78)$. Cette croyance se manifeste, dans le texte, à travers d'abord la disparition de l'oued de Djaffar et le rétrécissement de l'espace de la population indigène, qui subit la politique de la spoliation des terres :

L'oued a disparu et mes jardins et mes moulins. Ils ne nous ont rien laissé. Ils ont tous pris. C'était notre terre et nous l'aimions. Nous l'aimions depuis que Djaffar, le premier, y avait élevé sa maison et que ses fils, l'un après l'autre l'avait remise avec respect à celui qui devait la recevoir. Mais moi, j'en étais privé, on me l'a volé, sans elle je suis perdue, je ne sais plus que tresser des paniers inutiles et maudire le diable dont la face grimace sur la porte de cet immeuble $(2002: 21)$.

Le grand-père d'Aïcha souffre de l'usurpation dont il est victime. L'immeuble construit par madame Boissier s'offre à son regard comme étant le symbole ostentatoire de cette dépossession : "Nous avions deux moulins, là où s'élève aujourd'hui cette façade aux masques hideux» (2002: 21). Par ailleurs, la narratrice suggère, dès le début, que cet espace n'a plus sa raison d'être: "Il avait eu ses heures de gloires, le vieil immeuble de la rue en pente» (2002: 7). Le recours au plus que parfait et l'utilisation de l'adjectif «vieil» suggèrent très fortement que l'immeuble est désormais de trop dans son emplacement. Ainsi, dès l'entame, le lecteur sait que cet espace est voué à disparaître, tandis que l'oued est appelé à réapparaître : « Les fumés des voitures ont noirci les vieux murs et les petits anges joufflus semblent avoir de la peine à soutenir les balcons. Le vent, la pluie, la poussière et le temps ont effacé leurs sourire » (2002: 123).

L'immeuble dont madame Boissier a tant rêvé subit les outrages du temps et la maltraitance de la société moderne. Sa lente dégradation représente, à l'échelle microcosmique, la détérioration progressive du Monde : « trop usé pour réagir, il se livrait à cette force nouvelle qui ne le tourmentait pas encore mais l'affaiblissait chaque jour un peu plus»(2002: 73). À mesure que l'oued lutte pour revoir la lumière, l'immeuble s'affaiblit, laisse tomber des corniches, s'acheminant inexorablement vers la destruction. Cette prévalence des forces naturelles sur les forces cultivées, du «saltus " sur la «domus», nous incite à qualifier l'immeuble d'espace liminaire ${ }^{3}$ : "L'oued devra agir seul.Il ne saurait tarder.Il a tant fait pour revoir la lumière. L'architecte, l'entrepreneur, tous sont aveugles. Comment peuvent-ils penser que quelques travaux superficiels pourront faire du vieil immeuble de la rue en pente un immeuble neuf?» (2002: 118).

Selon Scarpa, " la mort est la forme la plus radicale du saltus » (2009: 212); or, la quasi majorité des termes désignant l'immeuble ou se rapportant à lui relèvent du champ 
lexical de la mort: "l'immeuble fané, recroquevillé » (33), »le vieil immeuble se laissait ronger " (73), "Le vieil immeuble n'avait plus sa raison d'être» (94), " L'immeuble agonisant » (115). À maintes reprises, l'immeuble est assimilé à une vieille personne, mais cette personnification permet seulement de le doter d'un corps, et d'un corps mourant. En d'autres termes, l'immeuble n'est « animé » que pour mieux être montré « inanimé » : «Vois-tu, les vieilles maisons sont comme les vieilles personnes. Elles n'aiment pas être brusquées, elles ne supportent pas être bousculées, elles n'ont plus assez de forces ni pour résister, ni pour lutter et le pire, c'est que parfois elles n'en ont plus envie » (2002: 87).

Si l'immeuble n'est pas véritablement doté d'un effet de vie ou d'une volonté propre, c'est parce qu'il doit s'envisager non pas seulement comme personnage dans le roman, mais comme allégorie de la mort. De fait, nous pensons qu'Un oued, pour la mémoire figure un combat entre la vie (représentée par l'oued) et la mort (qu'incarne l'immeuble), avec la volonté de faire triompher la vie : «Comment avait-il pu se résoudre à couler son béton sur un oued vivant?» (2002:23).

Malgré sa façade délabrée et ses murs lézardés, Aïcha considère le vieil immeuble de la rue en pente comme un héritage précieux. C'est que, pour elle, cet espace représente la mémoire des siens : "L'immeuble de la rue en pente était son immeuble. Grand-père le lui avait légué par-delà le temps. Que lui importe les sarcasmes des autres, leur mine triste et leurs soupirs exagérés! 'Cet immeuble! J'y ramasse ma vie ! avait-elle parfois envie de dire, mais vous ne pouvez pas comprendre!"'" (2002: 59). Dans la mesure où il est construit sur l'oued de Djaffar, » l'immeuble démodé» (59) garde le souvenir de la première maison de la ville dont il est la réplique. C'est en ce sens qu'il acquiert également une dimension mythique. En se le réappropriant, Aïcha se rend justice, tout en se faisant légataire d'une mémoire ancestrale: «Le vieil immeuble de la rue en pente n'avait plus qu'elle pour se souvenir et Aïcha réalisa combien désormais elle allait être seule » (2002: 67).

Vieille et malade, Aïcha vit en parfaite symbiose avec son immeuble. La relation qui s'établit entre son corps et les murs de celui-ci est comparable à celle qu'établit Balzac entre madame Vauquer et sa pension:

Ce qu'elle redoutait pour ses murs était dans sa poitrine. Une douleur pointue comme un zigzag tranchant, de la ceinture à l'épaule, le temps d'un soupir qui se bloque dans la gorge et ces battements fous d'un cœur qui s'emballe, un brouillard épais derrière les paupières lourdes et puis plus rien... $(2002: 81)$.

L'immeuble a une fonction spéculative. Tel un miroir, il renvoie à Aïcha l'image de son intériorité perturbée, de son Moi éclaté : » il semble que l'image de la maison devienne la topographie de notre être intime » écrit Bachelard (1957 : 27). Le personnage s'en va donc en même temps que son espace, ce dont il a d'ailleurs parfaitement conscience: "L'immeuble s'en allait, tout doucement, il s'en allait et c'est tout ce que Aïcha ressentait vraiment» (2002:112).

Mais Aïcha est surtout "un[e] rêve[euse] des voix souterraines, des voix étouffées et lointaines » (Bachelard, $1948:$ 167) : "je sais écouter et je sais rêver » (57). En s'enfermant dans son havre de paix, elle capte les bruits issus des eaux souterraines de l'oued :

Elle n'aurait pas le temps de retrouver l'oued. Il était là pourtant, tout près. Chaque nuit, le tumulte de ses flots impatients parvenait à l'oreille attentive. Dans sa rumeur sourde se mêlaient des voix: celle puissante de Djaffar, celle douce de grand-père, celle triste du docteur, et toutes les autres encore, les conquérantes, les vengeresses, les laborieuses, les triomphantes, les radieuses de tous ceux qui avaient bu son eau. Aïcha les distinguait toutes ces voix (2002:119).

15 Véritable lieu de mémoire, l'oued charrie la voix des ancêtres si chère à Aïcha ainsi que celle de tous ceux qui ont bu de son eau: "l'eau [...] remet en vie tous les souvenirs» 
affirme Bachelard (1942 : 93). Sa résurgence, vers la fin du texte, confirme sa pérennité ainsi que son importance, en tant que lieu de mémoire : "Les lionceaux avaient disparu, les moulins ne seraient plus, le vieil immeuble allait les rejoindre mais l'oued toujours en garderait le souvenir. Grand-père le comprenait déjà ainsi_» (2002: 108).

\section{Le déluge et la résurgence de l'oued}

La croyance dans la destruction et la recréation périodique de l'Univers implique l'idée que l'Âge d'or ne se trouve pas uniquement dans un passé mythique, mais aussi dans un avenir fabuleux (Eliade, 1975 : 70). Autrement dit, dans la doctrine indienne qui retient notre attention, l'Âge d'or est recouvrable, répétable. Mais, comme nous l'avons déjà souligné, la possibilité de réintégrer la perfection initiale demande l'anéantissement total de tout ce qui a existé et, partant, s'est dégradé depuis la création du Monde. Dès lors, la question qui se pose est la suivante : comment la Fin du Monde est-elle amenée dans la société du texte?

Disons tout de suite que l'attente d'un nouvel Âge d'or est très manifeste dans le texte. Mais, madame Boissier ne voulait pas croire que son immeuble s'effondrera un jour :

Madame Boissier, avertie du danger qu'il y avait peut-être à bâtir son immeuble à cet endroit, reprocha à son architecte de vouloir retarder les travaux [...].

- Quel risque ! Voulez-vous m'expliquer ? lui avait-elle demandé avec agacement.

- Et bien, s'il y avait réellement un oued au-dessous, ses eaux peuvent grossir après de fortes pluies par exemple et venir ronger les fondations et...

- Monsieur ! l'avait-elle interrompu, d'abord rien ne prouve qu'il y a ici un oued, et si oued il y a,je ne le crains pas! Voilà trente ans que je vis dans ce pays et les oueds, je les connais! Quant aux pluies diluviennes que vous redoutez, je n'en ai jamais vues! À supposer même, que chaque année l'oued fantôme vienne lécher les solides fondations que vous m'avez promises, combien de temps faudrait-il pour que l'immeuble s'effondre?

-Cent ans, peut-être moins, on ne peut savoir vraiment...

Madame Angèle Boissier était partie d'un éclat de rire qui fit trembler l'architecte de colère retenue devant cette femme un peu vulgaire mais qui payait si bien (2002 : 9).

17 Cet extrait met en évidence l'attitude méprisante qu'affichent les usurpateurs, à savoir la famille Boissier, vis-à-vis de l'histoire du pays colonisé. La fonction émotive du langage y tient une place importante et offre le spectacle d'une femme totalement aveuglée par son rêve et gouvernée par lui : «Madame Boissier était partie d'un éclat de rire " précise la narratrice. Cet entêtement à vouloir faire fi de la mémoire du lieu est une guerre contre les racines. Et le nom patronymique, » Boissier ", programme déjà cette lecture, puisqu'il signifie « escroc » : «C'était notre terre et nous l'aimions [...] Mais moi j'en ai été privé, on me l'a volée » (2002:21).

Angèle a le goût des richesses et le souci des apparences :

L'immeuble de la rue en pente était d'abord né dans le cœur d'Angèle. Elle le rêvait cossu et chaleureux, avec de hautes fenêtres et des balcons graciles, des corniches ouvragées, des anges en pierre joufflus, des gouttières déversant leurs eaux par des gueules de lions ou peut-être bien des tigres, des rosaces, des mascarons, enfin tous ces petits détails qui ne trompent pas, pensait-elle, sur la qualité du propriétaire (2002: 8-9).

18 La physionomie de l'immeuble est à l'image du personnage qui l'habite et entre dans sa caractérisation même: "Dis-moi la maison que tu imagines, je te dirai qui tu 
es» (Durand, $1983:$ 277). L'ironie de la narratrice sollicite la complicité du lecteur et provoque son rire, du fait de la discordance introduite entre le souci de l'élégance et le mauvais goût mis en avant. La puissance de l'illusion que se donne Angèle perce dans l'abondance des détails architecturaux et l'emploi des verbes "rêver" et " penser ». Mais, plus généralement, l'ironie de la narratrice s'éclaire d'un retour en arrière. Car Madame Boissier est une ancienne détenue accusée de vol :

On lui avait dit qu'elle était libre à condition de ne pas retourner en France et on lui avait indiqué l'adresse d'une pension pour dames modestes où elle aurait le temps de se préparer à affronter son nouveau destin. C'est sans doute pour toutes ces raisons que Madame Angèle Boissier tenait tant à avoir une vue sur la mer. Elle ne l'avait jamais traversée la mer ! Et la France pour elle n'était plus que le souvenir d'un gigantesque crassier, lugubre et menaçant, dont l'immensité bleue la séparait et la protégeait $(2002: 8)$.

Madame Boissier fait construire l'immeuble de son rêve pour qu'il reflète son nouveau statut. Mais environs cent ans après, les pluies diluviennes auxquelles elle ne croyait pas eurent lieu, conformément à la prophétie de son architecte :

Les pluies diluviennes que madame Angèle Boissier ignorait, auxquelles elle ne croyait pas, eurent pourtant lieu.

Ce fut un hiver comme on n'en avait jamais vu ou alors depuis longtemps, une tempête venue de tous les horizons. La mer se gonfla et se joua des digues. Les palmiers qui s'offraient aux rivages se tordirent et certains, épuisés par la lutte, se couchèrent en travers des routes inondées. La montagne disparut dans les nuages noirs et toutes les sources, même celles dont on ne se souvenait plus, rejaillissent aux endroits les plus inattendus (2008:123).

Cette description est tout à fait éloquente : un tel spectacle évoque l'anéantissement du vieux monde par le "Déluge». Les termes et les expressions employés ("pluies diluviennes », » un hiver comme on n'en a jamais vu », » tempête venue de tous les horizons ", » la mer gonfla », " routes inondées ", «montagne disparue », " nuages noirs ») mettent en lumière la puissance destructrice de la nature toute entière. Ce motif de la destruction par les eaux, associé au motif complémentaire de la régénération («toutes les sources, même celles dont on ne se souvenait plus, rejaillissent aux endroits les plus inattendus ») est fondamental dans Un oued, pour la mémoire: pour que l'oued de Djaffar puisse rejaillir, il faut que le vieux monde soit complètement anéanti.

Dans cet ordre d'idée, la terrible inondation qui s'abat sur la ville est à la fois dévastatrice et rénovatrice. Ces extraits en témoignent :

[...] toutes les sources, même celles dont on ne se souvenait plus, rejaillissent aux endroits les plus inattendus (2002:123).

Et dans la ville rassérénée, à petits pas, l'une emmenait l'autre en promenade. Elles ne parlaient pas beaucoup au début, elles flânaient par les rues animées, hésitant à la croisée des chemins (2002: 124).

Après le retour du beau temps, la ville est joie, chaleur et lumière. L'idée de recréation s'exprime par les termes 《rejaillissent », » rassérénée », » animées ». Mounia est l'incarnation de ce renouveau: "Mounia était là, revenue pour un temps, et Aïcha, le cœur gonflé, découvrait chaque jour sur le visage juvénile cette lumière qui parfois exaltait le regard, creusait des zones d'ombre, annonçait l'avenir (2002 : 123-124, Nous soulignons). La dédramatisation est également assurée à travers le retour au temps heureux de l'enfance et la mise en avant des sentiments d'apaisement qu'animent Aïcha : 
Aïcha avait confiance. Comme au temps de grand-père. La voie était retrouvée. Mounia la portait en elle comme grand-père l'avait jadis portée "Grand-mère avait dit Mounia, le beau temps est revenu, nous devrions sortir un peu... » [...] - Où veux-tu que je t'emmène ? Interrogeait la jeune fille. - Marchons ! répondit Aïcha, on verra bien! Mais toujours, quel que soit le détour, leurs pas les conduisaient vers cette rue en pente... (2002:124).

Les comparaisons établies dans ce passage (" comme...comme ») et le caractère répété de la scène - Aïcha se promène avec sa petite-fille, le long de la rue en pente, comme jadis avec son grand-père - confèrent ouvertement à l'œuvre une dimension circulaire. Cette circularité est en parfaite adéquation avec la thématique de l'éternel retour, que renforcent la résurgence de l'oued et l'effondrement du vieil immeuble :

L'oued a tenu ses promesses. Une nuit de printemps, le vieil immeuble s'est penché sur cette rue en pente dans un salut étrange et puis il a semblé s'agenouiller dans un craquement sourd. [...]

Aïcha a pris son voile et s'est glissée le long des rues vers la pente de son histoire.

Son cœur bat très fort et ses doigts se crispent sur les pans de ce voile qui lui échappe pour la première fois.

La côte est dure à gravir et ses halètements lui brouillent même la vue. Encore un pas et puis un autre, l'immeuble est là, l'immeuble n'est pas là. Mais il y a grandpère, là-haut, sur le talus couvert de broussailles. Ce n'est pas le vieillard courbé sur sa canne d'olivier. Il est beau, il est grand, il est jeune. Son burnous est jeté négligemment sur ses épaules. C'est grand-père et c'est Djaffar et il rit, il est heureux. Il lui montre l'oued au-delà du talus (2002 : 125-126).

Ce passage a tout d'un récit eschatologique : l'effondrement de l'immeuble agonisant représente, à l'échelle microcosmique, la destruction du vieux Monde, tandis que la résurgence de l'oued implique l'idée de son renouvellement et du retour des morts. Cette recréation coïncide avec l'avènement $d u$ "printemps ", saison porteuse d'un symbolisme régénérateur évident. Enfin, on ne peut manquer de percevoir l'importance symbolique du retour des morts, puisque, selon de vieilles croyances mythiques, lorsque "le Cosmos sera détruit et recréé, [...] la tribu recouvrera une sorte de Paradis: les morts ressusciteront, et il n'y aura ni mort ni maladie" (Eliade, 1975: 90). Ainsi, la réapparition de Djaffar et d'un grand-père «beau » et «jeune», la résurgence de l'oued ainsi que la régression, pour Aïcha, dans le temps heureux de l'enfance équivalent à une réactualisation des Temps mythiques de l'origine, donc à une re-création du Monde.

En guise de conclusion, nous pouvons dire que l'analyse des lieux de mémoire, chez Bakhaï, montre que celle-ci s'adonne, dans son roman, à une véritable poétique de l'espace. Le recours aux mythes d'origine et eschatologique, le déploiement massif des figures de rhétorique, l'établissement d'un jeu de correspondances entre les personnages et l'espace qu'ils occupent, l'écriture circulaire et la sollicitation fréquente de la mémoire individuelle et collective en sont les aspects les plus intéressants.

Ces procédés scripturaux indiquent aux lecteurs que l'espace est au fondement de l'univers fictionnel qui n'existerait pas sans lui. Loin de fournir le seul cadre de l'intrigue, il est la matière romanesque même et la visualisation des enjeux mémorielles et symboliques du récit 


\section{BIBLIOGRAPHIE}

Enfin, nous croyons avoir démontré que le mythe eschatologique structure la fiction. Mais il remplit également une fonction sociopolitique importante : en situant l'Âge d'or à la fois dans le passé et dans l'avenir, Bakhaï offre à ses concitoyens aux prises avec les affres de la guerre civile, l'espérance de la restauration de la paix et de la justice, dans un futur plus ou moins proche.

ABDOUN, Ismaïl, Lecture(s) de Kateb Yacine, Casbah Éditions, Alger, 2006.

BACHELARD, Gaston, L'Eau et les rêves. Essai sur l'imagination de la matière, José Corti, Paris, 1956 [1942].

BACHELARD, Gaston, La Terre et les rêveries du repos, José Corti, Paris, 1948. Disponible sur [http : //classiques.uqac.ca], (Consulté le 01/06/2019).

BACHELARD, Gaston, Poétique de l'espace, PUF, Paris, 1957. Disponible sur : [http : $/$ classiques.uqac.ca], (Consulté le 25/03/2019).

BAKHAÏ, Fatéma, Un oued, pour la mémoire, Dar El Gharb, Oran, 2002 [1995].

BAKHAÏ, Fatéma, » Les Algériens et leur Histoire », in Résolang, Numéro spécial, Novembre 2012, pp. 7-10. Disponible sur : [http://sites.univ-lyon2.fr/résolang/], (consulté le 30/5/2019).

BÉNÉJAM-BONTEMPS, Marie-Josette, » Âge d'or », dans BRUNEL PIERRE (dir.), Dictionnaire des mythes littéraires, Éditions du Rocher, Monaco, 1988, pp. 52-56.

BENRAMDANE, Farid, » De l'étymologie de Wahran : de Ouadaharan à Oran », in Insaniyat, $\mathrm{N}^{\circ} 23-24$, janvier - juin 2004, pp. 249-272. Disponible sur [https://www.asjp.cerist.dz/ en/article/43040], (Consulté le 15/06/ 2019).

DURAND, Gilbert, Les Structures anthropologiques de l'imaginaire, Dunod, Paris, 1983 [1969].

ELIADE, Mircea, La Nostalgie des origines, Gallimard, Paris, 1971 [1969].

ELIADE, Mircea, Aspects du mythe, Gallimard, Paris, 1975 [1963].

ELIADE, Mircea, Le Sacré et le Profane, Gallimard, Paris, 1988 [1965].

MOKHTARI, Rachid, Le Nouveau Souffle du roman algérien. Essai sur la littérature des années 2000, Chihab Éditions, Alger, 2006.

NORA, Pierre, Les lieux de mémoire : I. La République, Gallimard, Paris, 1984.

SCARPA, Marie, L'Eternelle Jeune fille. Ethnocritique du Rêve de Zola, Honoré Champion, Paris, 2009.

STORA, Benjamin, La Guerre invisible: Algérie, années 1990, Presses des Sciences Po, Paris, 2001.

STORA, Benjamin, La Guerre des mémoires. La France face à son passé colonial, Éditions de l'Aube, La Tour d'Aigues, 2007.

\section{NOTES}

1. Cet article reprend sous forme synthétique les résultats partiels de recherches menées dans le cadre de notre thèse de doctorat consacrée à la poétique de l'espace dans la littérature algérienne francophone contemporaine, sous la direction du Prof. Assia Kacedali à l'université Alger 2. 
2. L'édition que nous utilisons, en l'occurrence Dar El Gharb (2002), ne comporte aucune indication générique.

3. Dans son ouvrage intitulé L'Eternelle jeune fille. Une ethnocritique du Rêve de Zola, Marie Scarpa déclare qu' « une lecture anthropologique des lieux du roman, selon un axe domestique / sauvage, paraît fort fructueuse et en mesure d'enrichir les études que l'on en fait dans la narratologie traditionnelle » (2009: 207). Ce type d'étude s'appuie sur la «tripartition [la domus/le campus/le saltus], que les historiens et les géographes de la ruralité empruntent aux Romains et que les ethnologues se sont mis à utiliser aussi. La domus se définirait, très rapidement, comme l'espace familier (là où demeure la famille au sens large), le lieu de vie et de la vie; le campus, le lieu de la production, du travail ; le saltus comme [...] la prévalence des forces naturelles sur les forces cultivées mais aussi de l'irrationnel sur la raison, du monde invisible sur le monde visible : en ce sens, le rêve, la rêverie, le sens de l'inconnu, l'au-delà en relèvent » ( 2009 : 208-209).

\section{RÉSUMÉS}

Cette étude porte sur la poétique des lieux de mémoire chez Fatéma Bakhaï. Elle s'inspire essentiellement des travaux de Mircea Eliade, en envisageant les espaces mis en texte comme des espaces sacrés et, partant, significatifs et symboliques. Ces espaces sont également dépositaires de vécus et de souvenirs collectifs et contribuent, de fait, à recréer l'Histoire. Enfin, le recours aux mythes d'origine et eschatologique constitue le moyen par lequel l'auteure réfléchit à la réalité sociale, en produit une critique, voire en annonce les changements.

This study focuses on the poetics of space in Un oued, pour la mémoire by Fatéma Bakhaï. Drawing on the works of Mircea Eliade, it considers the spaces described in the text sacred spaces and, consequently, significant and symbolic. These spaces are also repositories of experiences and collective memories which contribute to recreating History. Finally, the use of original and eschatological myths is a means through which the author reflects on social reality, produces criticism, and even announces changes.

\section{INDEX}

Mots-clés : espace, mémoire, Histoire, mythe, eschatologie

Keywords : space, memory, History, myth, eschatology

\section{AUTEUR \\ BRAHIM AIT AMOKRANE}

Université Alger 2, Algérie 